\title{
JOACHIM WACH*
}

\section{Bibliography}

Kitagawa, Joseph M., Heiler, Friedrich, and Neumann, Käthe, "Bibliographie Joachim Wachs," in: Joseph M. Kitagawa, Gibt es ein Verstehen fremder Religionen? ("Joachim Wach-Vorlesungen der Theologischen Fakultät der Philipps-Universität Marburg/Lahn”, Vol. I). Leiden, E. J. Brill, 1963, pp. 32-36.

“Bibliography of Joachim Wach (1922-55)," in: Joseph M. Kitagawa, ed., Understanding and Believing. Essays by Joachim Wach (Harper Torchbooks). New York and Evanston, Harper \& Row, 1968, pp. 188-196.

Bibliography in: Archives de Sociologie des Religions, I, 1 (janvier-juin 1956), pp. 64-69.

\section{Biography and Appreciation}

Benz, E., "Bericht über das Werk von Joachim Wach: 'Types of Religious Experience, Christian and non-Christien', London 1951," Zeitschrift fiir Religions- und Geistesgeschichte, IX (1957), pp. 371-374.

Bolle, Kees W., "Wach's legacy: reflexions on a new book," History of Religions, $\mathrm{X}, 1$ (August 1970), pp. 80-90.

Desroche, H., "Sociologie et théologie dans la typologie religieuse de Joachim Wach," Archives de Sociologie des Religions, I, 1 (janvier-juin 1956), pp. 41-63.

Heiler, Friedrich, "Joachim Wach" (Memorial Address), The Divinity School News (University of Chicago), XXII, 4 (November 1955), pp. 28-32.

French translation: "Souvenirs sur Joachim Wach," Archives de Sociologie des Religions, I, 1 (janvier-juin 1956), pp. 21-24.

Kitagawa, Joseph M., "A glimpse of Professor Wach," The Chicago Theological Seminary Register, XLV, 4 (November 1955).

-, "Joachim Wach" (Memorial Address), The Divinity School News (University of Chicago), XXII, 4 (November 1955). Reprinted in: Joseph M. Kitagawa, ed., Understanding and Believing. Essays by Joachim Wach. (Harper Torchbooks). New York and Evanston, Harper \& Row, 1968. pp. 197-201. 
-, "Joachim Wach and Sociology of Religion," The Journal of Religion, XL, 3 (July 1957).

French translation: "Joachim Wach et la sociologie de la religion," Archives de Sociologie des Religions, I, 1 (janvier-juin 1956). pp. 25-40.

-.., "The life and thought of Joachim Wach," in: Joachim Wach, The Comparative Study of Religions. New York, Columbia University Press, 1958, pp. XIII-XLVIII.

A Dutch translation "Het leven en denken van Joachim Wach" appeared in the Dutch edition of the book: Joachim Wach, Vergelijkende godsdienstwetenschap (Aula 218). Utrecht-Antwerpen, Het Spectrum, 1965, pp. 9-41.

A German translation of this introduction appeared in the German edition of this book: Joachim Wach, Vergleichende Religionsforschung. (Urbanbücher, 52). Stuttgart, Kohlhammer, 1962, pp. 10-34.

-, "Joachim Wach, Leben, Forschung und Lehre," in: Joseph M. Kitagawa, Gibt es ein Verstehen fremder Religionen? Leiden, Brill, 1963, pp. 1-31.

-, "Gibt es ein Verständnis fremder Religionen? Zur Religionspsychologie und Religionssoziologie von Joachim Wach," in: Joseph M. Kitagawa, Gibt es ein Verstehen fremder Religionen? Leiden, Brill, 1963, pp. 37-66.

-, "Introduction" in: Joseph M. Kitagawa, ed., Understanding and Believing. Essays by Joachim Wach. ("Harper Torchbooks"). Evanston and Now York, Harper \& Row, 1968, pp. VII-XVIII.

-, "Verstehen and Erlösung: some remarks on Joachim Wach's Work," History of Religions, XI, 1 (August 1971), pp. 31-53.

Klimkeit, Hans-Joachim, "Das Prinzip des Verstehens bei Joachim Wach," Numen, Vol. XIX, Fasc. 2-3 (July-December, 1972), pp. 216-228.

Poniatowski, Zygmunt, ,Joachim Wach," Euhemer. Przeglad Religioznaw, zy, 1958, Nr. 2, pp. $39-45$.

-, "Joachim Wach jako socjolog religii," in: J. Wach, Socjologia religii. Warszawa, Wstęp, 1960, pp. IX-XXIX.

Rudolph, Kurt, "Joachim Wach (1898-1955)," in: Bedeutende Gelehrte in Leipzig. Leipzig, 1965, pp. 229-237.

Séguy, Jean, "Joachim Wach, sociologue des religions," Archives de Sociologi des Religions, 7e Année, No 14 (juillet-décembre 1962), pp. 27-34.

Werblowsky, R. J. Zwi, "The Comparative Study of Religions - a Review Essay," Judaism, Vol. 8, No. 4 (Fall 1959), pp. 1-9.

Scheimann, Richard, W., Wach's Theory of the Science of Religion. Ph. D. Diss. Univergity of Chicago, 1963.

Schoeps, Hans-Joachim, "Joachim Wachs wissenschaftliche Bedeutung," Zeitschrift für Religions- und Geistesgeschichte, IX (1957), pp. 368-371.

\section{Method and Theory}

1923 “Bemerkungen zum Problem der 'extremen' Würdigung der Religion," Zeitschrift für Missionskunde und Religionswissenschaft (Berlin), XXXVIII (1923), pp. 161-183.

1923 "Zur Methodologie der allgemeinen Religionswissenschaft," Zeitschrift für Missionskunde ..., XXXVIII (1923).

1924 Religionswissenschaft: Prolegomena zu ihrer wissenschaftstheoretischen Grundlegung. Leipzig, J. C. Hinrichs. 
1924 “" 'Nur'. Gedanken über den Psychologismus," Zeitschrift für Missionskunde ..., XXXIX (1924), pp. 209-215.

1925 “Wilhelm Dilthey über 'Das Problem der Religion'," Zeitschrift für Missionskunde ..., XXXX (1925), pp. 66-81.

1926-1933 Das Verstehen: Grundzüge einer Geschichte der hermeneutischen Theorie im 19. Jahrhundert, 3 vols. Tübingen, Mohr.

1927 "Jakob Burckhardt und die Religionsgeschichte," in: Die Religion in Geschichte und Gegenwart, 2nd edition, Vol. I, 1927, pp. 1270-1271.

1929 "Die Geschichtsphilosophie des 19. Jahrhunderts und die Theologie der Geschichte", Historische Zeitschrift, Nr. 142 (1929), pp. 1-15.

1929 "Und die Religionsgeschichte?," Zeitschrift für Systematische Theologie (Gütersloh), VI (1929), pp. 484-497.

1929 "Idee und Realität in der Religionsgeschichte," Zeitschrift für Theologie und Kirche (Tübingen), (1927?), pp. 334-364.

1930 "Religionsphilosophie", "Religionssoziologie", "Religionswissenschaft," in: Religion in Geschichte und Gegenwart, 2nd edition, Vol. IV, 1930.

1930 "Zur Hermeneutik heiliger Schriften," Theologische Studien und Kritiken (Stuttgart and Gotha), CII (1930), pp. 280-290.

1931 "Verstehen," in: Die Religion in Geschichte und Gegenwart, 2nd edition, Vol. V, 1931, pp. 1570-1573.

1931 "Religionssoziologie," in: A. Vierkandt, ed,, Handwörterbuch der Soziologie. 1931, pp. 479-494.

1931 "Das religiöse Gefühl," in: Das Problem der Kultur und die ärztliche Psychologie. Vorträge des Instituts der Medizin an der Universität Leipzig, Nr. 4, Leipzig, Thieme, 1931, pp. 9-33 (idem, 1932).

1934 "Religiöse Existenz," Zeitschrift für Missionskunde ..., XXXIX (1934), pp. 193-201.

1935 "Sinn und Aufgabe der Religionswissenschaft," Zeitschrift für Missionskunde..., Band L, Heft 5 (1935), pp. 133-147.

English translation: "The Meaning and Task of the History of Religions (Religionswissenschaft)" in: J. M. Kitagawa, ed., with the coll. of Mircea Eliade and Charles H. Long, The History of Religions: Essays on the Problem of Understanding. Chicego, University of Chicago Press, 1967, pp. 1-17. Reprinted in: Understanding and Believing. (Harper Torchbooks). New York, Harper \& Row, 1968, pp. 125-145.

1937 "Der Begriff des Klassischen in der Religionswissenschaft," in: Quantulacunque. Studies presented to Kirsopp Lake. London, Christophers, 1937, pp. 87-97.

English translation "The concept of the 'classical' in the study of religions," in: Joachim Wach, Types of Religious Experience. Christian and nonChristian. Chicago, Un. of Chicago Press, 1951, pp. 48-57.

1939 "Religionssoziologie," in: S. R. Steinmetz, ed., Gesammelte kleine Schriften zur Ethnologie und Soziologie, pp. 479-494.

1945 "Sociology of Religion," in: Georges Gurvitch, Twentieth Century Sociology. New York, Philosophical Library, pp. 405-437.

1946 "On Understanding," in: A. A. Roback, ${ }^{\text {dd., The Albert Schweitzer }}$ Jubilee Book. Cambridge (Mass.), SCI-ART Publishers, pp. 131-146.

1947 "The Place of the History of Religions in the Study of Theology," Journal of Religion, XXVII, 3 (July 1947), pp. 157ff. Reprinted in: Types of Religious Experience, Christian and non-Christian. Chicago, University of Chicago Press, 1951. 
1950 "On Teaching History of Religions," in: Pro Regno pro Sanctuario [Festschrift, G. van der Leeuw]. Nijkerk (Holland), Callenbach.

1952 "Radhakrishnan and the comparative study of religion," in: Paul A. Schilpp, ed., The Philosophy of Sarvepalli Radhakrishnan. New York, Tudor Publishing Co., pp. 443-458.

1953 "Rudolph Otto und der Begriff des Heiligen," in: Arnold Bergsser, ed., Deutsche Beiträge. Chicago, Henry Regnery Co., pp. 200-217.

1954 "General Revelation and the Religions of the World," Journal of Bible and Religion, XXII, 2 (April 1954), pp. 83 ff.

\section{Main Publications}

1922 Der Erlösungsgedanke und seine Deutung. (Veröffentlichungen des Forschungsinstituts für vergleichende Religionsgeschichte an der Universität Leipzig, 8). Leipzig, J. C. Hinrichs.

1925 Mahayana, besonders im Hinblick auf das Saddharma-Pundarika-Sutra: eine Untersuchung über die religionsgeschichtliche Bedeutung eines heiligen Textes der Buddhisten. München-Neubiberg, Schloss.

1925 Meister und Jünger: zwei religionsgeschichtliche Betrachtungen. Tübingen, J. C. B. Mohr.

1931 Einführung in die Religionssoziologie. Tübingen, J. C. B. Mohr.

1932 Typen religiöser Anthropologie: ein Vergleich der Lehre vom Menschen im religionsphilosophischen Denken von Orient und Okzident. Tübingen, J. C. B. Mohr.

1934 Das Problem des Todes in der Philosophie unserer Zeit. Tübingen, J. C. B. Mohr.

1935 "Eine neue katholische Philosophie der Religionsgeschichte," Zeitschrift für Missionskunde . . ., L (1935), pp. 375-385.

1935 J. Wach, ed., Religion und Geschichte. Heft I, Stuttgart, Kohlhammer.

1944 Sociology of Religion. Chicago, University of Chicago Press. Also London, Kegan Paul, 1947.

French translation by M. Lefevere: Sociologie de la religion. Paris, Payot, 1954.

German translation: Religionssoziologie, by Helmut Schoek, based on the 4th e dition. Tübingen, J. C. B. Mohr, 1951.

Polish translation with introduction by Zygmunt Poniatowski: Socjologia religii. Warszawa, Wstęp, 1960.

1951 Types of religious experience, Christian and non-Christian. Chicago, University of Chicago Press. Collection of papers. For 'Method and Theory' see especially "Universals in the History of Religion" and "The concept of the 'classical' in the Study of Religions." Fifth impression, pb., 1972.

1958 The Comparative Study of Religions. Edited with Introduction by Joseph M. Kitagawa. New York, Columbia University Press.

German translation: Vergleichende Religionsforschung. (Urban Bücher). Stuttgart, W. Kohlhammer Verlag, 1962.

Spanish translation: El Estudio Comparado de las Religiones. Buenos Aires, Editorial Paidos, 1967.

1968 Kitagawa, Joseph M., ed., Understanding and Believing. Essays by Joachim Wach (Collection of 17 papers with Bibliography and In Memoriam). New York-Evanston, Harper. 


\section{CLEMENT C. J. WEBB}

\section{Bibliography}

Religious Experience. A public Lecturs delivered on 19 May 1944 by C. C. J. Webb. Printed together with a bibliography of his published writtings [by L. W. Grensted], and presented to him by some of his Friends and Pupils on the Occasion of his 80th Birthday. London, Oxford University Press, 1945, pp. 49-70.

\section{Main Publications}

1908 The Notion of Revelation. (Pan-Anglican Papers S. B. 5). London, Society for Promoting Christian Knowledge.

1912 Natural and Comparative Religion. An insugural Lecture. Oxford, Clarendon Press.

1915 Studies in the History of Natural Theology. Oxford, Clarendon Press.

1916 Group Theories of Religion and the Individual. London, Allen \& Unwin; New York, Macmillan.

1933 Religion and Theism. The Forwood Lectures delivered at Liverpool University. Together with a chapter on the psychological Accounts of the Origin of Belief in God. London, Allen \& Unwin, Oxford University Press.

1935 The historical Elements in Religion. (Lewis Fry Lectures 1934). London, Allen \& Unwin.

\section{MAX WEBER*}

\section{Bibliography}

Weber, Marianne, “Bibliographie," in: Marianne Weber, Max Weber. Ein Lebers. bild. Tübingen, Mohr, 1926, pp. 715-719.

Prades, J. A., "Liste chronologique des travaux de Max Weber," in: J. A. Prades, La sociologie de la religion chez Max Weber. Louvain - Paris, Nauwelaerts et Béatrice-Nauwelaerts, 19692, pp. 259-271.

\section{Biography and appreciation}

Adams, L. J., "'The Protestant Ethic' with fewer tears," in: In the Name of Life. Essays in Honor of Erich Fromm. New York, Holt, Rinehart and Winston, 1971, pp. 174-190.

Aron, R., "Introduction," in: Max Weber, Le savant et le politique. Paris, Plon, 1959, pp. 34-57.

-, Les grandes doctrines de sociologie historique. Vol. II: Emile Durkheim, Vilfredo Pareto, Max Weber. Paris, C. D. U., 1962. 
Baumgarten, Edward, "Einleitung," in: J. Winckelmann, ed., Max Weber, Soziologie, Weltgeschichtliche Analysen, Politik. Stuttgart, Kröner Verlag. $1960^{2}$, pp. XI-XXVI.

Max Weber. Werk und Person. Dokumente auggewählt und kommentiert. Tübingen, Mohr, 1964.

Bendix, Reinhard, Max Weber. An. Intellectual Portrait, New York, Doubleday, 1960 .

German translation by Renate Rausch: Max Weber. Das Werk. Darstellung. Analyse. Ergebnisse. Preface René König. München, Piper, 1964.

Bendix, Reinhard, and Roth, Guenther, Scholarship and Partisanship. Essays on Max Weber. Berkeley-Los Angeles-London, University of California Press, 1970.

Birnbaum, N., "Conflicting interpretations of the rise of capitalism: Marx and Weber," British Journal of Sociology, IV (1953), pp. 125-141.

Bosse, Hans, Marx, Weber, Troeltsch. Religionssoziologie und marxistische Ideologie Kritik. München, Kaiser Verlag; Mainz, Grünewald Verlag, 1970.

Bourdieu, P., "Une interprétation de la théorie de la religion selon Max Weber," Archives Européennes de Sociologie, XII, 1 (1971), pp. 3-21.

Brann, H. W., "Max Weber and the United States," Southwestern Social Science Quarterly (June, 1944), pp. 18-30.

Dux, G., "Religion, Geschichte und sozialer Wandel in Max Webers Religionssoziologie," Internationales Jahrbuch für Religionssoziologie, Vol. 7. KölnOpladen, 1971, pp. 60-94.

Eisenstadt, S. N., "The Protestant Ethic thesis in an analytical and comparative framework," in S. N. Eisenstadt, ed., The Protestant Ethic and Modernization. New York, Basic Books, 1968.

Fechner, E., "Der Begriff des kapitalistischen Geistes bei Sombart und Max Weber," Weltwirtschaftliches Archiv (1929), pp. 194-211.

Fischler, K. H., "Kritische Beiträge zu Prof. M. Webers Abhandlungen: Die protestantische Ethik," Archiv für Sozialwissenschaft und Sozialpolitik, XXV, 1 (1907), pp. 232-242.

Fischoff, Ephraim, "The Protestant Ethic and the Spirit of Capitalism: the history of a controversy," Social Research, II (February, 1944), pp. 53-77. Reprinted in: Robert W. Green, ed. Protestantism and Capitalism: the Weber Thesis (Problems in European Civilization). Boston, Heath, 1959, pp. 107-114.

Freund, J. and Luthy, H., "Controverse sur Max Weber," Preuves, 163 (1964), pp. 85-92.

Freund, Julien, "L'éthique économique et les religions mondiales selon Max Weber," Archives de Sociologie des Religions, $13^{e}$ Année, No 26 (juilletdécembre 1968), pp. 3-25.

Max Weber ("Philosophes"). Paris, P. U. F., 1969.

Gerth, H. H. and Gerth, H. I., "Bibliography on Max Weber," Social Research, XVI (1949), pp. 70-98.

Gerth, H. H. and Mills, C. Wright, "Introduction: the man and his work," in: H. H. Gerth and C. Wright Mills, From Max Weber: Essays in Sociology. Translated, edited and with an Introduction. New York, Oxford University Press, 1946, pb. 1958, pp. 1-74.

Goldschmidt, D., "Bericht über die Verhandlungen zum Rahmenthema: Die Aktualität Max Webers in der modernen Religionssoziologie," in: O. Stam. 
mer, ed., Max Weber und die Soziologie heute. Verhandlungen des 15. deutschen Soziologentages. Tübingen, Mohr, 1965, pp. 221-246.

Gordon-Walker, P. C., "Capitalism and the Reformation," Economic History Review, VIII, 1 (1937), pp. 1-19.

Gouldner, Alvin W., "On Weber's analysis of bureaucratic rules," in: R. K. Merton, ed., Reader in Bureaucracy. Glencoe (Ill.), Free Press, 1952.

Green, Robert W., ed., Protestantism and Capitalism: The Weber Thesis and its Critics. (Problems in European Civilization). Boston, Heath, 1959.

Halbwachs, Maurice, "Economistes et historiens: Max Weber, un homme une cuvre," Annales d'Histoire Economique et Sociale, No 1 (janvier 1929).

Henrich, D., Die Einheit der Wissenschaftslehre Max Webers. Tübingen, Mohr, 1952.

Honingsheim, P., "Max Weber. His religious and ethical background and development," Church History, XIX, 4 (Dec. 1950).

Jaspers, Karl, Gedenkrede (Max Weber), Heidelberg, 17. Juli 1920. In print 1921.

-, Max Weber. Deutsches Wesen im politischen Donken, im Forschen und Philosophieren, 1932. New revised edition under the title of Max Weber. Politiker, Forscher, Philosoph. München, Piper Verlag, 1946, 1948, 1958.

Kolko, G., "Max Weber on America," History and Theory (The Hague). I, 3 (1961), pp. 243-260.

König, René, and Winckelmann, Johannes, eds., Max Weber zum Gedächtnis. Materialien und Dokumente zur Bewertung von Werk und Persönlichkeit (Kölner Zeitschrift für Soziologie und Sozialpsychologie, Sonderheft 7). Köln-Opladen, 1963.

Kosa, J., and Rachiele, L. D., "The spirit of capitalism, traditionalism and religiousness: A re-examination of Weber's concepts," Sociological Quarterly, IV, 3 (1963), pp. 243-269.

Lennert, R., Die Religionstheorie Max Webers. Diss. Leipzig, 1931; in print 1935. Lopes, J. R., "Max Weber," Sociologica, XVIII, 1 (1956), pp. 51-69.

Löwith, Karl, "Max Weber und Karl Marx," Archiv für Sozialwissenschaft und Sozialpolitik, LXVII, 1 (1932), pp. 53-99 and 175-214.

Mitzman, Arthur, The Iron Cage. An Historical Interpretation of Max Weber. New York, Knopf, 1970.

Mommsen, Wolfgang, Max Weber und die deutsche Politik 1890-1920. Tübingen, Mohr, 1959.

Moth, G. and Bendix, R., "Max Webers Einfluss auf die amerikanische Soziologie," Kölner Zeitschrift für Soziologie und Sozialpsychologie, XI (1959), pp. 38-53.

Oppenheimer, H., Die Logik der soziologischen Begriffsbildung mit besonderer Berücksichtigung von Max Weber. Tübingen, Mohr, 1925.

Palyi, Melchior, and Schulze-Gaevernitz, Gerhart von, and others, Erinnerungsgabe für Max Weber, 2 vols. München, 1923.

Parsons, Talcott, "The author and his career," in: Max Weber, The Theory of Social and Economic Organization. Glencoe (Ill.), Free Press, 1947, pp. 1-5.

-, "Translator's Preface," in: Max Weber, The Protestant Ethic and the Spirit of Capitalism. Translated by Talcott Parsons. London, 1930. Pb. New York, Charles Scribner's Sons, 1958, pp. IX-XI.

-, "Preface to New Edition," in: Max Weber, The Protestant Ethic and the Spirit of Capitalism. Translated by Talcott Parsons. Pb. New York, Charles Scribner's Sons, 1958, pp. XIII-XVII. 
-, "Introduction," in: Max Weber, The Sociology of Religion. Translated by Ephraim Fischoff. Boston, Beacon Press, pb. 1964, pp. XIX-LXVII.

-, "Wortgebundenheit und Objektivität in den Sozialwissenschaften. Eine Interpretation der Beiträge Max Webers," in: P. Stammer, ed., Max Weber und die Soziologie heute. Verhandlungen des 15. deutschen Soziologentages. Tübingen, Mohr, 1965, pp. 39-64.

Paul, R. S., "Weber and Calvinism: the effects of a'calling'," Canadian Journal of Theology, XI, 1 (1965), pp. 25-4l.

Prades, J. A., La sociologie de la religion chez Max Weber. Louvain - Paris, Nauwelaerts et Béatrice-Nauwelaerts, 1969².

Rheinstein, Max, Max Weber on Law in Economy and Society. Cambridge, Harvard University Press.

Robertson, H. M., Aspects of the Rise of Economic Individualism. A Criticism of Max Weber and his School. New York, Kelley and Millman, 1933, 1959".

Salman, D. H., "Psychology and sociology in Weber's theories," Social Compass, X, 6 (1963), pp. 536-539.

Salmon, J. H. M., "Religion and economic motivation: some French insights on an old controversy," Journal of Religious History, II, 2 (1963), pp. 181-203.

Samuelsson, Kurt, Ekonomi och religion. 1957.

English translation by E. Geoffrey French: Religion and Economic Action.

$A$ Critique of Max Weber. New York, Harper Torchbooks, 1964.

French translation: Économie et religion. Une critique de Max Weber. Introduction par D. C. Coleman. (L'Euvre sociologique, 2). ParisThe Hague, Mouton, 1971.

Schåf, J. J., Geschichte und Begriff. Eine kritische Studie zur Geschichtsmethodologie von E. Troeltsch und M. Weber. 1946.

Schelting, Alexander von, Die Wissenschaftslehre Max Webers. Tübingen, 1934.

Schill, B., Die Rezeption von M. Webers Lehre des sozialen Handelns durch Talcott Parsons. Diss. München, 1964.

Schnipper, L., "Max Weber on the sociological basis of the Jewish Religion," Jewish Journal of Sociology, I, 2 (1959), pp. 250-260.

Shils, Edward A., "Foreword" in: Edward A. Shils and Henry A. Finch, tr. and ed., Max Weber, On the Methodology of the Social Sciences. Glencoe (Ill.), Free Press, 1949.

Sprenkel, O. B. van der, "Max Weber on China," History and Theory (The Hague), III, 3 (1964), pp. 348-370.

Starnmer, O., ed., Max Weber und die Soziologie heute. Verhandlungen des 15. deutschen Soziologentages. Tübingen, Mohr, 1965.

Stark, W., "Capitalism, calvinism and the rise of modern science," Sociological Review, XLIII, 5 (1951), pp. 95-104.

-, “Mex Weber's sociology of religious belief," Sociological Analysis, XXV, 1 (1964), pp. 41-49.

Steeman, T. M., "Max Weber's sociology of religion," Sociological Analysis, XXV, 1 (1964), pp. 50-58.

Strötgen, J., Zur Literatur über M. Webers Aufsatz "Die protestantische Ethile und der Geist des Kapitalismus," Un. Köln, Soziologisches Seminar, 1960-1961.

Tawney, R. H., "Foreword" in: Max Weber, The Protestant Ethic and the Spirit of Capitalism. Translated by Talcott Parsons. London, 1930. Pb. New York, Charles Scribner's Sons, 1958, pp. 1 (a)-1 (e)-11. 
Tellegen, E., De sociologie in het werk van Max Weber. Meppel, Boom Pers, 1968.

Tenbruck, F., "Die Genesis der Methodologie Max Webers," Kölner Zeitschrift für Soziologie und Sozialpsychologie, XI (1959), pp. 573-630.

Thorner, I., "Ascetic protestantism and the development of science and technology," American Journal of Sociology, LVIII, 1 (1952), pp. 25-33.

Turksma, L., "Protestant ethic and rational capitalism: a contribution to a never ending discussion," Social Compass, IX, 5-6 (1962), pp. 445-473.

Weber, Marianne, Max Weber. Ein Lebensbild. Tübingen, Mohr, 1926; Heidel berg, $1950^{2}$.

-, Lebenserinnerungen. Bremen, Storm, 1948.

Weber, Max, "Politische Briefe", in: Gesammelte Politische Schriften. München, Drei Masken, 1921 , pp. 451-488.

-, Jugendbriefe 1876-1893. Edited by Marianne Weber. Tübingen, Mohr, n. d. (1936).

Weinrich, M., Max Weber, l'homme et le savant. Paris, Vrin, 1938.

Winckelmann, Johannes, Legitimität und Legalität in Max Webers Herrschaftssoziologie. Mit einem Anhang: Max Weber, "Die drei Typen der legitimen Herrschaft." Tübingen, Mohr, 1952.

\section{Method and Theory}

1904 "Die Objektivität sozialwissenschaftlicher und sozialpolitischer Erkenntnis," Archiv für Sozialwissenschaft und Sozialpolitik, XIX (1904), pp. 22-87. Reprinted in Gesammelte Aufsätze zur Wissenschaftslehre, Tübingen, Mohr, 1924, pp. 146-201.

Spanish translation: "La objetividad del conocimiento propio de las ciencias sociales y de la política social," Revista de Economía Política, VII/2-3 (1956), pp. 423-490.

1906 "Kritische Studien auf dem Gebiet der kulturwissenschaftlichen Logik. I: Zur Auseinandersetzung mit Eduard Meyer. II: Objektive Möglichkeit und adëquate Verursachung in der historischen Kausalbetrachtung," Archiv für Sozialwissenschaft und Sozialpolitik, XXII (1906), pp. 143-207, Reprinted in Gesammelte Aufsätze zur Wissenschaftslehre, Tübingen Mohr, 1924, pp. 215-290.

1909 "Energetische Kulturtheorien," Archiv für Sozialwissenschaft und Sozıalpolitik, XXIX (1909), pp. 575-598. Reprinted in Gesammelte Aufsätze zur Wissenschaftslehre, Tübingen, Mohr, 1924, pp. 376-402.

1913 "Utber einige Kategorien der verstehenden Soziologie," Logos, IV (1913), pp. 253-294. Reprinted in Gesammelte Aufsätze zur Wissenschaftslehre, Tübingen, Mohr, 1924, pp. 403-450.

1917 "Der Sinn der Wertfreiheit der soziologischen und ökonomischen Wissenschaften," Logos, VII (1917), pp. 40-88. Reprinted in Gesammelte Aufsätze zur Wissenschaftslehre, Tübingen, Mohr, 1924, pp. 451-502.

1924 Gesammelte Aufsātze zur Wissenschaftslehre (Collected essays). Edited by Marianne Weber. Tübingen, Mohr. $1951^{2}$ (expanded; edited by J. Winckelmann).

Partial English translation by Edward A. Shils and Henry A. Finch, with a Foreword by Edward A. Shils: Max Weber, On the Methodology of the Social Sciences. Glencoe (Ill.), Free Press, 1949. 
French translation of part of the book with introduction by Julien Freund: Essais sur la thérie de la science. Paris, Plon, 1965.

Partial Italian translation by $\mathrm{P}$. Rossi: Il metodo delle scienze storicosoziali. Torino, Einaudi, 1958.

1960 Soziologische Grundbegriffe. Tübingen, Mohr.

English translation: Basic Concepts in Sociology. New York, The Philosophical Library, 1962.

1968 Methodologische Schriften. Studienausgabe. Edited with an introduction by Johannes Winckelmann. Frankfurt am Main.

\section{Main Publications}

1889 Zur Geschichte der Handelsgesellschaften im Mittelalter. Nach südeuropäischen Quellen. Stuttgart. Amsterdam, 1964.

1891 Die römische Agrargeschichte in ihrer Bedeutung für das Staats- und Privatrecht. Stuttgart. Amsterdam, 1962.

Italian translation: La storia agraria romana. Milano, Societd Editrice Libraria, 1907.

1892 Die Verhältnisse der Landarbeiter im ostelbischen Deutschland, dargestellt auf Grund der vom Verein für Sozialpolitik veranstalteten Erhebungen. Leipzig.

1895 Der Nationalstaat und die Volkswirtschaftspolitik (Inaugural Address). Freiburg i. Br. Reprinted in Gesammelte Politische Schriften, München, Drei Masken, 1921, pp. 7-30.

1896 "Die sozialen Gründe des Untergangs der antiken Kultur," Die Wahrheit, VI (1896), pp. 57-77. Reprinted in Gesammelte Aufsätze zur Sozial- und Wirtschaftsgeschichte. Tübingen, Mohr, 1924, pp. 289-311.

English translation by C. Mackauer: "The social causes of the decay of ancient civilization," The Journal of General Education (Chicago), $\mathrm{V}$ (1950), pp. 75-88.

Spanish translation: "La decadencia de la cultura antigua," Revista de Occidente, XIII (1926), pp. 25-59.

1905 Die protestantische Ethik und der Geist des Kapitalismus. First published in Archiv für Sozialwissenschaft und Sozialpolitik, XX (1905), pp. 1-54, and XXI (1905), pp. 1-110. Then published separately. Reprinted in Gesammelte Aufsätze zur Religionssoziologie, Vol. I. Tübingen, Mohr, 1920, pp. 1-236.

Reprinted many times. New edition: Die protestantische Ethik. Edited by Johannes Winckelmann, Vol. I: Eine Aufsatzsammlung. Vol. II: Kritiken und Antikritiken. München-Hamburg, Siebenstern-Taschenbuch, 2 vols. 1965-1968.

English translation by Talcott Parsons: The Protestant Ethic and the Spirit of Capitalism. With a foreword by R. H. Tawney. London and New York, 1930, 1948, 1950. Pb. New York, Scribner, 1958.

French translation by Jacques Chavy: L'éthique protestante et l'esprit du capitalisme. Suivi d'unun autre essai. Paris, Plon, 1964.

Italian translation by P. Burresi: Ftica protestante e lo spirito del capitalismo. Roma, Leonardo, 1945.

Spanish translation: La ética protestante y el espiritu del capitalismo. Madrid, Editorial Revista Derecho Privado, 1955. 
1906 "Die protestantischen Sekten und der Geist des Kapitalismus," Frankfurter Zeitung (13 und 14 April 1906). Reprinted in Gesammelte Aufsätze zur Religionssoziologie, Vol. I. Tübingen, Mohr, 1920.

1908 "Kritische Bemerkungen zu H. K. Fischers Aufsatz, "Kritische Beiträge zu Mex Webers Abhandlung 'Die protestantische Ethik und der Geist des Kapitalismus' "," Archiv für Sozialwissenschaft und Sozialpolitik, XXV (1908), pp. 243-249.

1908 “'Bemerkungen zu der 'Replik' von H. K. Fischers Protestantische Ethik und Geist des Kapitalismus,' Archiv für Sozialwissenschaft und Sozialpolitik, XXVI (1908), pp. 275-283.

1908-1909 "Zur Psychophysik der industriellen Arbeit," Archiv für Sozialwissenschaft und Sozialpolitik, XXVII (1908), pp. 730-770; XXVIII (1909), pp. 219-277, 719-761; XXIX (1909), pp. 513-542. Reprinted in Gesammelte Aufsätze zur Soziologie und Sozialpolitik. Tübingen, Mohr, 1914, pp. 61-255.

1910 “Antikritisches zum 'Geist' des Kapitalismus," Archiv für Sozialwissenschaft und Sozialpolitik, XXX (1910), pp. 176-202.

1910 “Antikritisches Schlusswort zum 'Geist' des Kapitalismus," Archiv für Sozialwissenschaft und Sozialpolitik, XXXI (1910), pp. 554-599.

1914 Gesammelte Aufsätze zur Soziologie und Sozialpolitik (Collected essays). Tübingen, Mohr.

1916-1919 "Die Wirtschaftsethik der Weltreligionen. Einleitung. I: Konfuzianismus und Taoismus. Zwischenbetrachtung: Theorie der Stufen und Richtungen religiöser Weltablehnung. II: Hinduismus und Buddhismus. TII: Das antike Judentum," Archiv für Sozialwissenschaft und Sozialpolitik, XLI (1916), pp. 1-87; 335-421; 613-744; XLII (1916/17) pp. 345-461; 687-814; XLIV (1917), pp. 52-138; 349-443; 601-626; XLVI (1919), pp. 40-113; 311-366; 541-604. Reprinted in Gesammelte Aufsütze zur Religionssoziologie. Tübingen, Mohr, 1920, Vol. I, pp. 237-573, Vol. IJ, Vol. III, pp. 1-400. For the English translations, see under 19201921.

1917 "Die P'harisäer" (manuscript), in Gesammelte Aufsätze zur Religions. soziologie, Tübingen, Mohr, 1920, Vol. III, pp. 401-442.

1919 Wissenschaft als Beruf. München, Duncker und Humblot. Reprinted in Gesammelte Aufsätze zur Wissenschaftslehre. Tübingen, Mohr, 1922, pp. $524-555$.

English translation: "Science as a vocation" in From Max Weber: Essays in Sociology (1946, pb. 1958, 1964), pp. 129-156.

French translation by Julien Freund in: Max Weber, Le savant et le politique. Introduction by Raymond Aron. Paris, Plon, 1959; Paris, Union générale d'éditions, 1963.

Italian translation: Il lavoro intellettuale come professione. Torino, Einaudi, 1948.

1919 Politik als Beruf. München, Duncker und Humblot. Reprinted in Gesammelte politische Schriften. München, Drei Masken, 1921, pp. 396-450; Berlin, 1964.

English translation: "Politics as a vocation" in: From Max Weber: Essays in Sociology (1946, pb. 1958, 1964), pp. 129-156.

French translation by Julien Freund in: Max Weber, Le savant et le politique. Introduction by Raymond Aron. Paris, Plon, 1959; Paris, Union générale d'éditions, 1963. 
1920-1921 Gesammelte Aufsätze zur Religionssoziologie, 3 vols. Tübingen, Mohr. Vol. I: "Die protestantische Ethik und der Geist des Kapitalismus", "Die protestantischen Sekten und der Geist des Kapitalismus", "Die Wirtschaftsethik der Weltreligionen I: Einleitung, Konfuzianismus und Taoismus, Zwischenbetrachtung (Theorie der Stufen und Richtungen religiöser Weltablehnung)" (19474, 19635). Vol. II: “Die Wirtschaftsethik der Weltreligionen II: Hinduismus und Buddhismus" (1963', 1966"). Vol. III: "Die Wirtschaftsethik der Weltreligionen III: Das antike Judentum, Nachtrag (Die Pharisëer).” (1963³, 19664).

English translations:

Volume I was partly translated by Talcott Parsons in The Protestant Ethic and the Spirit of Capitalism (see above, under 1905). Part of it was translated by Hans $\mathrm{H}$. Gerth and C. Wright Mills in From Max Weber: Essays in Sociology (1946, pb. 1958, 1964), pp. 267-359. The third part of Volume One was translated separately by Hans H. Gerth: The Religion of China: Confucianism and Taoism. Pb. Glencoe, Ill., Free Press, 1951. A fragment of this can be found in From Max Weber: Essays in Sociology (1946, pb. 1958, 1964), pp. 416-444.

Volume II was translated by Hans H. Gerth and Don Martindale: "The Hindu social system," The University of Minnesota Sociology Club Bulletin, Nr. 1, 1950. It was reissued under the title of The Religion of India. The Sociology of Hinduiom and Buddhism. New York, The Free Press; London, Collier-Macmillan, 1958, pb. 1967. A fragment of this can be found in From Max Weber: Essays in Sociology (1946, pb. 1958, 1964), pp. 396-415.

Volume III was translated by Hans H. Gerth and Don Martindale: Ancient Judaism. Pb. Glencoe (Ill.), Free Press, 1952.

French translation: Etudes de sociologie de la religion. Vol. I, Part I: L'éthique protestante et l' esprit du capitalisme. Translated by Jacques Chavy. Paris, Plon, 1964.

1921 "Die Stadt. Eine soziologische Untersuchung," Archiv für Sozialwissenschaft und Sozialpolitik, XLVII ( 92 ), pp. $62-772$. Reprinted in Gesammelte Aufsätze zur Sozial- und Wirtschaftsgeschichte. Tübingen, Mohr, 1924, pp. 54-60.

English translation by Don Martindale and Gertrude Neuwirth: The City. Glencoe (Ill.), Free Press, 1958. Pb. New York-London, Free Press, 1966. Italian translation: La cittd. Milano, Bompiani, 1950.

1921 Die rationalen und soziologischen Grundlagen der Musik. Published with an introduction by Theodor Kroyer. München, 1924². Reprinted in Wirtschaft und Gesellschaft. Tübingen, Mohr, 1922, pp. 818-869.

English translation by Don Martindale, Johannes Riedel and Gertrude Neuwirth: The Rational and Social Foundations of Music. Southern Illinois University Press, 1958.

1921 Gesammelte politische Schriften. Tübingen, Mohr. New edition by Johannes Winckelmann, with a preface by Theodor Heuss, $1958^{2}$.

1922 “Die drei Typen der legitimen Herrschaft," Preussische Jahrbücher, 187 (1922), pp. 1-12. Reprinted in Johannes Winckelmann, Legitimität und Legalität in Max Webers Herrschaftssoziologie. Tübingen, Mohr, 195\%. English translation by H. H. Gerth: "The three types of legitimate rule," Berkeley Publications in Society and Institutions, IV, 1 (1958).

Italian translation: Carismatica e $i$ tipi del podere. Torino, Unione, 1934. 
1922 Wirtschaft und Gesellschaft. Grundriss der verstehenden Soziologie. Tübingen, Mohr, 1922, 1925², 19473. A new revised edition, edited by Johannes Winckelmann, appeared in 2 vols., Tübingen, Mohr, 1956. A study edition appeared in Köln-Berlin, 1964 ( 2 vols.). This study contains "Religionssoziologie", which was also published separately: Tübingen, Mohr, 1922.

The following books contain English translations of parts of Wirtschaft und Gesellschaft:

1946 From Max Weber: Essays in Sociolugy. Translated and edited by Hans H. Gerth and C. Wright Mills. New York, Oxford University Prese, 1946; pb. 1958; London, 1948, 1952, 1967. (pp. 159-266).

1947 The Theory of Social and Economic Organization. Translated by A. M. Henderson and Talcott Parsons, with an Introduction and Annotations by T. Parsons. London-New York, Oxford University Press, 1947, 1950. Pb. Glencoe (Ill.), Free Press, 1957.

1954 On Law and Economy in Society. Translated by Edward Shils and Max Rheinstein. Edited with Introduction and Notes by Max Rheinstein (20th Century Legal Philosophy Series, 6). Cambridge (Mass.), Harvard University Press, 1954.

1958 The Rational and Social Foundations of Music. Translated and edited by Don Martindale, Johannes Riedel and Gertrude Nouwirth. Southern Illinois University Press, 1958.

1961 Theories of Society. Edited by Talcott Parsons, Edward Shils, Kaspar D. Naegele, Jesse R. Pitts, 2 vols. Glencoe (Ill.), Free Press, 1961. 1963 The Sociology of Religion. Translated by Ephraim Fischoff, with an Introduction by Talcott Parsons. Boston, Beacon Press, 1963, pb. 1964; London, Social Science Paperbacks, 1966.

1968 On Charisma and Institution Building. Selected Papers. Edited with an Introduction by S. N. Eisenstadt. Chicago-London, University of Chicago Press, 1968. Phoenix pb. 1968.

Italian translation: Economia e societd. Milano, Communita, 1962.

Spanish translation by J. M. Echavarria and others: Economia y saciedad. 4 vols. México, Fondo de cultura económica, 1944.

1922 Gesammelte Aufsätze zur Wissenschaftslehre. See above, under '3. Method and Theory'.

1923 Wirtschaftsgeschichte. Abriss der universalen Sozial- und Wirtschaftsgeschichte. Edited by S. Hellmann and M. Palyi. München, Mohr. 1924², $1958^{3}$ (expanded and edited by Johannes Winckelmann).

English translation by Frank H. Knight: General Economic History. New York-London, George Allen and Unwin, 1927; Glencoe (Ill.), Free Press, 1950.

Spanish tranglation by Manuel Sánchez Sarto: Historia económica general. México, Fondo de cultura económica, 1942, $1956^{2}$.

1924 Gesammelte Aufsatze zur Sozial- und Wirtschaftsgeschichte. Edited by Marianne Weber. Tübingen, Mohr.

1924 Gesammelte Aufsätze zur Soziologie und Sozialpolitik. Edited by Marianne Weber. Tübingen, Mohr.

1956 Soziologie, Weltgeschichtliche Analysen, Politik. Edited and annotated by Johannes Winckelmann. Introduction by Eduard Baumgarten. Stuttgart, Kröner. 
1956 Staatssoziologie. Soziologie des rationalen Staates und der modernen polntischen Parteien und Parlamente. Edited with Inuroduction by Johannes Winckelmann. Tübingen, Mohr.

1960 Rechtssoziologie. Edited from the manuscript, with Introduction, by Johannes Winckelmann (Soziologische Texte, 2). Neuwied, H. Luchterhand, 1960; Berlin, 1966².

\section{FRIEDRICH G. WELCKER}

\section{Main Publications}

1844-1867 Kleine Schriften, 5 vols. Bonn, Weber.

1857-1862 Griechische Götterlehre, 3 vols. Göttingen, Dietrich.

\section{JULIUS WELLHAUSEN*}

\section{Bibliography}

Alfred Rahlfs, "Verzeichnis der Schriften Julius Wellhausens," (Studien zur semitischen Philologie und Religionsgeschichte Julius Wellhausen zum siebzigsten Geburtstag am 17. Mai 1914 gewidmet von Freunden und Schülern und in ihrem Auftrag herausgegeben von Karl Marti), Beihefte zur Zeitschrift für die Alttestamentliche Wissenschaft, XXVII (1914), pp. 351-368.

\section{Biography and Appreciation}

Baumgartner, W., "Wellhausen und der heutige Stand der alttestamentlichen Wissenschaft," Der Theologische Rundschau, N. F. II (1930), pp. 287-307.

Baxter, W. L., Sanctuary and Sacrifice. A Reply to Wellhausen. London, Eyre, 1896.

Becker, C. H., "Julius Wellhausen," Der Islam, IX (1918), pp. 95-99. Reprinted in: C. H. Becker, Islamstudien. Vom Werden und Wesen der islamischen Welt, Vol. II. Berlin, 1932, pp. 474-480.

Boschwitz, Fr., Julius Wellhausen. Motive und Maszstäbe seiner Geschichtsschreibung. Diss. Phil., Marburg, 1938.

Cohen, H., "Julius Wellhausen. Ein Abschiedsgrusz," Jüdische Schriften, Vol. II. Berlin, 1924, pp. 463-468.

Greszmann, H., "Julius Wellhausen,” Protestantenblatt, 1918, Nr. 6.

Jepsen, A. "Wellhausen in Greifswald," Festschrift zur 500-Jahrfeier der Universität Greifswald, Vol. II, 1956, pp. 47-56.

Perlitt, Lothar, Vatke und Wellhausen. Geschichtsphilosophische Voraussetzungen und historiographische Motive für die Darstellung der Religion und Geschichte 
Israels durch Wilhelm Vatke und Julius Wellhausen. Berlin, A. Töpelmann, 1965.

Schwartz, Eduard, "Julius Wellhausen," Nachrichten von der Königlichen Gesellschaft der Wissenschaften zu Göttingen. Geschäftliche Mitteilungen aus dem Jahre 1918, (Berlin) 1918, pp. 43-70. Reprinted in: Eduard Schwartz, Gesammelte Schriften, Vol. I. Berlin, 1938, pp. 326-361.

Wellhausen, Julius, "Wellhausens Briefe aus seiner Greifswalder Zeit (18721879) an den anderen Heinrich Ewald-Schüler Dillmann," edited by E. Barnikol. Wissenschaftliche Zeitschrift der Universität Halle, Ges. Sprachw., VI, 5 (1957), pp. 701-712. Reprinted in: Gottes ist der Orient. Festschrift für Otto Eissfeldt. Berlin, 1959, pp. 28-39.

\section{Main Publications}

1870, De gentibus et familiis Judaeis quae 1. Chr. 2.4 enumerantur (Theol. Liz.Diss.). Göttingen.

1871 Der Text der Bücher Samuelis untersucht. Göttingen, Vandenhoeck \& Ruprecht.

1874 Die Pharisäer und die Sadducäer. Eine Untersuchung zur inneren jüdischen Geschichte. Greifswald. Reprinted Hannover, Lafaire, 1924.

1876-1877 "Die Composition des Hexateuchs," Jahrbücher für Deutsche Theologie: 1876, pp. 392-450 and 531-602; 1877, pp. 407-479. Reprinted as Skizzen und Vorarbeiten II. Die Composition des Hexateuchs. Berlin, Reimer, 1885. Reprinted in Die Composition des Hexateuchs und der historischen Bücher des Alten Testaments, Berlin, Reimer, 18892, $1899^{3}$. Reprinted Berlin, Walter de Gruyter \& Co, $1963^{4}$.

1878 Geschichte Israels. Vol. I. Berlin, Reimer, 1878. The following editions appeared under the title of Prolegomena zur Geschichte Israels. Berlin, Reimer, 18832, $1886^{3}$, $1895^{4}, 1899^{5}, 1905^{6}$; Reprinted Berlin-Leipzig, $1927^{6}$.

English translation by J. S. Black and Allan Menzies: Prolegomena to the History of Israel, with a Reprint of the Article "Israel" from the "Encyclopaedia Britannica." With a Preface by W. Robertgon Smith. Edinburgh, A. \& C. Black, 1885. Pb. New York, Meridian Books, 1957.

1880 Geschichte Israels. Printed as a manuscript in about twenty copies. Greifswald, Christmas 1880. A revised and enlarged edition appeared under the title "Abriss der Geschichte Israels und Juda's" in Skizzen und Vorarbeiten, Vol. I. Berlin, Reimer, 1884.

English translation, considerably enlarged, as article "Israel" in Encyclopaedia Britannica, 9th edition, Vol. XIII, pp. 396-431. This article was published separately under the title Sketch of the History of Israel and Judah. London, A. \& C. Black, $1891^{3}$. It was also reprinted as an appendix to Prolegomena to the History of Israel. Edinburgh, A. \& C. Black, 1885: New York, 1957.

The "Abriss der Geschichte Israels und Juda's" is a German expanded translation of the article "Israel" in the Encyclopaedia Britannica.

1882 Muhammed in Medina. Das ist Vakidi's Kitab al Maghazi in verkürzter deutscher Wiedergabe herausgegeben. Berlin, Reimer, 1882.

1883 "Mohammedanism. Part I: Mohammed and the first four caliphs," Encyclopaedia Britannica, 9th edition, Vol. XVI, pp. 545-565. 
1884-1899 Skizzen und Vorarbeiten. 6 vols. Berlin, Reimer.

I: 1. Abriss der Geschichte Israels und Juda's. 2. Lieder der Hudhailiten, arabisch und deutsch. 1884.

II: Die Composition des Hexateuchs. 1885.

III: Reste arabischen Heidentums. 1887. Second edition under the title of Reste arabischen Heidentums gesammelt und erläutert. 1897. Reprinted Berlin, de Gruyter, $1961^{3}$.

IV: 1. Medina vor dem Islam. 2. Muhammads Gemeindeordnung von Medina. 3. Seine Schreiben, und die Gesandtschaften an ihn. 1889.

V: Die kleinen Propheten übersetzt, mit Noten. 1892, 18932, $1898^{3}$. Reprinted Berlin, Walter de Gruyter \& Co, $1963^{4}$.

VI: 1. Prolegomena zur ältesten Geschichte des Islams. 2. Verschiedenes. 1899.

1885 "Pentateuch and Joshua," Encyclopaedia Britannica, 9th edition, Vol. XVIII (1885), pp. 505-514. Reprinted as "Hexateuch," Encyclopaedia Biblica, Vol. II (1901), pp. 2045-2056.

1885 "Scholjen zum Diwan Hudail No. 139-280. Herausgegeben von Julius Wellhausen," Zeitschrift der Deutschen Morgenländischen Gesellschaft, 1885, pp. 411-480.

1886 "Septuagint," Encyclopaedia Britannica, 9th edition, Vol. XXI (1886), pp. 667-670.

1893 "Die Ehe bei den Arabern," Nachrichten von der Königlichen Gesellschaft der Wissenschaften zu Göttingen, 1893, pp. 431-481.

1893 "The Babylonian exile," The New World, II (1893), pp. 601-611.

1894 Israelitische und jüdische Geschichte. Berlin, Reimer. 1895², 18973, 19014, $1904^{5}, 1907^{6}, 1914^{7}$. Reprinted Berlin, 19589.

1895 The Book of Psalms. (Part 14 of "The Sacred Books of the Old Testament, A critical edition of the Hebrew text printed in colors, with notes", under the editorial direction of Paul Haupt). Translation of the notes by J. D. Prince. Leipzig, Hinrichs; Baltimore, The Johns Hopkins Press; London, David Nutt, 1895.

1895 "Der syrische Evangelienpalimpsest vom Sinai," Nachrichten von der Königlichen Gesellschaft der Wissenschaften zu Göttingen, Phil.Historische Klasse, pp. 1-12.

1895 "Die Rückkehr der Juden aus dem babylonischen Exil," Nachrichten von der Königlichen Gesellschaft der Wissenschaften zu Göttingen, Phil.Historische Klasse, pp. 166-186.

1898 The Book of Psalms. A new translation. With explanatory notes and an Appendix on the Music of the Ancient Hebrews (Part 14 of "The Sacred Books of the Old and New Testaments. A new English translation with explanatory notes and pictorial illustrations," edited with the assistance of H. H. Furness by P. Haupt). Translation of the psalms by H. H. Furness; translation of the notes by J. Taylor; translation of the appendix by J. A. Paterson. London, James Clarke; New York, Dodd, Mead, and Co. Reprinted Leipzig, Hinrichs, 1904.

1901 Die religiös-politischen Oppositionsparteien im alten Islam ("Abhandlungen der Königlichen Gesellschaft der Wissenschaften zu Göttingen,” Phil.Historische Klasse, Neue Folge, Band V, Nr. 2). Berlin, Weidmann.

1901 "Die Kämpfe der Araber mit den Romäern in der Zeit der Umajjaden," Nachrichten von der Königlichen Gesellschaft der Wissenschaften zu Göttingen, Phil.-Historische Klasse, pp. 414-447. 
1902 Das arabische Reich und sein Sturz. Berlin, Reimer. Reprinted Berlin, Walter de Gruyter \& Co, 1960².

English translation by Margaret Graham Weir: The Arab Kingdom and Its Fall. Calcutte, University of Calcutta, 1927 (with Index).

1903 Das Evangelium Marci übersetzt und erklärt. Berlin, Reimer. 19092.

1904 Das Evangelium Matthaei übersetzt und erklärt. Berlin, Reimer.

1904 Das Evangelium Lucae übersetzt und erklärt. Berlin, Reimer.

1905 Einleitung in die drei ersten Evangelien. Berlin, Reimer, $1911^{2}$.

1905 "Israelitisch-jüdische Religion," in: P. Hinneberg, ed., Die Kultur der Gegenwart (Teil I, Abt. IV: "Die christliche Religion mit Einschlusz der israelitisch-jüdischen Religion"). Berlin-Leipzig, Teubner, 1905, pp. $1-40$; 19092, pp. 1-4l.

1905 "Utber den geschichtlichen Wert des zweiten Makkabäerbuchs," Nachrichten von der Königlichen Gesellschaft der Wissenschaften zu Göttingen, Phil.-Historische Klasse, pp. 117-168.

1907 Erweiterungen und Anderungen im vierten Evangelium. Berlin, Reimer.

1907 „Noten zur Apostelgeschichte,” Nachrichten von der Königlichen Gesellschaft der Wissenschaften zu Göttingen, Phil.-Historische Klasse, pp. 1-21

1907 Analyse der Offenbarung Johannis (,Abhandlungen der Königlichen Gesellschaft der Wissenschaften zu Göttingen," Phil.-Historische Klasse, Neue Folge, Band IX, Nr. 4). Berlin, Weidmann.

1908 Das Evangelium Johannis. Berlin, Reimer.

1914 Kritische Analyse der Apostelgeschichte ("Abhandlungen der Königlichen Gesellschaft der Wissenschaften zu Göttingen," Phil.-Historische Klasse, Neue Folge, Band XV, Nr. 2). Berlin, Weidmann.

1965 Arundrisse zum Alten Testament. Edited by Rudolf Smend. München

\section{ARENT JAN WENSINCK}

\section{Bibliography}

In: A. J. Wensinck, Semietische Studiën ..., 1941, pp. 9-12.

\section{Biography and Appreciation}

Huizinga J., "Levensbericht van Arent Jan Wensinck (7 augustus 188219 september 1939),"Jaarboek der Koninklijke Nederlandsche Akademie van Wetenschappen (1939-1940), pp. 215-224.

Massignon, L., "A. J. Wensinck," Journal Asiatique, CCXXXIII (1941-1942) pp. 213-215.

Pedersen, J., “Arent Jan Wensinck," Acta Orientalia, XVIII (1940), pp. 161-163.

\section{Main Publications}

1908 Mohammed en de Joden te Medina. Dissertation. Leiden, Brill.

1911-1913 Legends of Eastern Saints, chiefly from Syriac sources, edited and partly 
translated. Vol. I: The story of Archelides; Vol. II: The Legend of Hilaria. Loiden, Brill.

1916 The ideas of the Western Semites concerning the navel of the earth. (Verhandelingen Koninklijke Akademie van Wetenschappen, afd. Letterkunde. N. R. XVII, no. 1). Amsterdam, J. Müller.

1919 Bar Hebraeus's Book of the Dove, together with some Chapters from his Ethikon. With an introduction, notes and registers. Leiden, Brill.

1921 Tree and bird as cosmological symbols in western Asia. (Verhandelingen Koninklijke Akademie van Wetenschappen, afd. Letterkunde). Amsterdam.

1922 "The Semitic New Year and the Origin of Eschatology," Acta Orientalia, I (1922), pp. 158-199.

1923 Mystic treatises by Isaac of Niniveh, translated from Bedjain's Syriac text with an introduction and registers. (Verhandelingen Koninklijke Akademie van Wetenschappen, afd. Letterkunde. N. R. XXIII, no. 1). Amsterdan.

1924 "Mohammed und die Propheten," Acta Orientalia, II (1924), pp. 168-198.

1925 The Second Commandment. (Mededeelingen Koninklijke Akademie van Wetenschappen, afd. Letterkunde, deel 59, serie A, no. 6). Amsterdam.

1927 A Handbook of Early Muhammedan Tradition, alphabetically arranged. Leiden, Brill. Reprinted 1971.

1932 The Muslim Creed, its Genesis and Historical Development. Cambridge, University Press. Reprinted London, Frank Cass \& Co., 1965.

1933 On the relation between Ghazäli's Cosmology and his Mysticism. (Mededeelingen Koninklijke Akademie van Wetenschappen, afd. Letterkunde, deel 75, serie A, no. 7). Amsterdam, Noord-Hollandse Uitgevers-Maatschappij.

1936 Les preuves de l'existence de Dieu dans la théologie musulmane. (Mededeelingen Koninklijke Akademie van Wetenschappen, afd. Letterkunde, deel 81, serie A, no. 2). Amsterdam, Noord-Hollandse Uitgevers-Maatschappij.

1936-1971 Concordance et Indices de la tradition musulmane... Par A. J. Wensinck, avec le concours de nombreux orientalistes, 6 vols. Leiden, Brill.

1940 La pensée de Ghazzālì. Paris, Adrien-Maisonneuve.

1941 Semietische studiën uit de nalatenschap van Prof. Dr. A. J: Wensinck (7 augustus 1882-19 september 1939). Leiden, Sijthoff.

\section{EDVARD A. WESTERMARCK}

\section{Biography and Appreciation}

Ginsberg, Morris, "The life and work of Edward Westermarck," in: Morris Ginsberg, Reason and Unreason in Society (Essays in Sociology and Social Philosophy, Vol. Two). London, Heinemann, 1947 (repr. 1956, 1960, 1965), pp. 61-83.

Hirn, Yrjö, "Edvard Westermarck and his English Friends," Transactions of the Westermarck Society (Gothenburg), I (1947), pp. 39-51. 
Lagerborg, Rolf, Edvard Westermarck och verken frän hans verkstad 1902-1939.

(Skrifter utgivna av Svenska Litteratursällskapet i Finlands natur og folk. Heft 97, No. 1). Helsingfors-Kфbenhavn, 1951.

Tenkku, Jussi, Westermarcks Definition of the Concept of the World. Copenhagen, 1962.

\section{Main Publications}

1891 The History of Human Marriage, 3 vols. London, Macmillan. 1894; $1901^{3} ; 1921^{5}$.

1906-1908 Origin and Development of the Moral Ideas, 2 vols. London, Macmillan. $1912^{2}$.

1914 Marriage Ceremonies in Morocco. London, Macmillan.

1926 Ritual and Belief in Morocco, 2 vols. London, Macmillan.

1927 Minnen ur mitt liv. Helsingsfors, Schilat.

English translation from the Swedish by Anna Barwell: Memories of My Life. London, Allon \& Unwin, 1929.

\section{ULRICH VON WILAMOWITZ-MOELLENDORF}

\section{Bibliography}

Wilamowitz-Bibliographie, 1868 bis 1929. Hrsg. von F. H. von Geertringen und G. Klaffenbach. Berlin, Weidemannsehe Buchhandlung, 1929.

\section{Biography and Appreciation}

Mommsen und Wilamowitz. Briefwechsel, 1872-1903. Ed. by Baron J. F. W. R. A. and Baroness Dorothea M. H. von Gaertringen. Berlin, 1935.

Ulrich von Wilamowitz-Moellendorf, Erinnerungen 1848-1914. Leipzig, Koehler, 1928.

Ulrich von Wilamowitz-Moellendorf. Dem Meister der Altertumswissenschaft zum 80. Geburtstage gewidmet. Berlin-Loipzig, 1929.

Usener und Wilamowitz. Ein Briefwechsel, 1870-1905. Ed. by Hermann Dietrich and Friedrich Hiller von Gaertringen. Leipzig-Berlin, 1934.

\section{Main Publications}

1901 Reden und Vorträge. 1902, $1913^{3}$ (expanded), 1925-19264 (revised and expanded, 2 vols.).

1921 Geschichte der Philologie. Einleitung in die Altertumewissenschaft. Leipzig.

1935 Kleine Schriften. Besorgt von Paul Maas, Edw. Schwartz, Ludolf Malten. Berlin. (Only Volumes 1 and 5 appeared). 


\title{
RICHARD WILHELM
}

\author{
Main Publications
}

1910 Kungfutse - Gespräche. (Translation). Jena. $1923^{2}$.

1910 Laotse - Das Buch des Alten vom Sinn und Leben. (Translation). Jena. $1923^{2}$.

1914 Chinesische Volksmärchen. Jena.

English translation by Frederick H. Martens: The Chinese Fairy Book.

Selected from Chinesische Volksmärchen. London, Fisher Unwin, 1922.

1923-1924 I Ging: Das Buch der Wandlungen, 2 vols. (Translation). Jena.

1925 Die Seele Chinas. Berlin, Hobbing.

English translation: The Soul of China. Text transl. by John Holroyd Reece, Poems by Arthur Waley. London, Jonathan Cape; New York, Harcourt, Brace and Co., 1928.

1925 Konfuzius.

English translation by George H. Danton and Annina Periam Danton: Confucius and Confucianism. New York, Harcourt, Brace and Co., 1931.

1925 Laotse und der Taoismus. (Frommanns Klassiker der Philosophie, Bd. 26). Stuttgart.

1925 Kung-Tse. Leben und Werk. (Frommanns Klassiker der Philosophie, Bd. 25). Stuttgart.

1928 Geschichte der chinesischen Kultur. München, 1928.

English translation by Joan Joshua: A Short History of Chinese Civilisation. Introduction by Lionel Giles. London, G. G. Harrap and Co.; New York, The Viking Press, 1929.

1931 Der Mensch und das Sein. (Vorträge und Aufä̈tze). Hrsg. von S. Wilhelm. Jena. $1939^{2}$.

1956 Wandlung und Dauer. Die Weisheit des I. Ging. First published in: Der Mensch und das Sein, 1931. Düsseldorf.

\section{GEORGE A. WILKEN}

\section{Bibliography}

Kinderen, T. H. der, "Lijst der in druk verschenen geschriften van Dr. G. A. Wilken," Bijdragen tot de Taal-, Land-en Volkenkunde van Nederlandsch Indië, Series V, Part 7 (1892), pp. 154-156.

Wilken, G. A., Verspreide Geschriften, 1912, Vol. I, pp. 18-24.

-, Opstellen over Adatrecht, 1926, pp. XXVII-XXX.

\section{Biography and Appreciation}

Goeje, M. J. de, "Levensbericht van G. A. Wilken," in: Jaarboek van de Koninklijke Akademie van Wetenschappen, 1892.

Kinderen, T. H. der, "Levensbericht van Dr. G. A. Wilken," Bijdragen tot de Taal-, Land en Volkenkunde van Nederlandsch Indie.. Series V, Part 7 
(1892), pp. 139-154. Reprinted in Verspreide Geschriften, 1912, Vol. I, pp. $3-17$.

Veth, P. J., "De ethnologische studiën van G. A. Wilken," Internationales Archiv für Ethnographie, IV (1891), pp. 282-284.

\section{Main Publications}

1885 Het Animisme bij de Volken van den Indischen Archipel. Amsterdam, De Bussy. First published in Indische Gids, VI and VII, 1884 and 1885.

1886-1887 "Utber das Haaropfer und einige andere Trauergebräuche bei den Völkern Indonesiens," Revue Coloniale Internationale, II (1886), pp. 225279; and III (1887), pp. 345-426.

1893 Handleiding voor de vergelijkende Volkenkunde van Nederlands Indië. Ed. by C. M. Pleyte. Leiden.

1912 Verspreide Geschriften, 4 vols. Ed. by Mr. F. D. E. van Ossenbruggen. Semarang-Soerabaja-The Hague, Van Dorp and Co.

1926 Opstellen over Adatrecht. Ed. by Mr. F. D. E. van Ossenbruggen. SemarangSoerabaja-Bandoeng-The Hague, Van Dorp and Co.

\section{HUGO WINCKLER}

\section{Bibliography}

"Winckler-Bibliographie" by O. Schroeder, in: Mitteilungen der Vorderasiatischen Gesellschaft, XX. 1 (1916), pp. 25-48.

\section{Main Publications}

1897-1905 Altorientalische F'orschungen, 3 vols. Leipzig.

1900 Geschichte Israels, Die Legende (Völker und Staaten des alten Orients, II; Die Geschichte Israels in Einzeldarstellungen). Leipzig, Pfeiffer.

1901 "Die altbabylonische Weltanschauung," Preussische Jahrbücher.

1901 Himmels- und Weltbild der Babylonier als Grundlage der Weltanschauung und Mythologie alter Völker. Leipzig. 1903².

1901 Arabisch-semitisch-orientalisch. Kulturgeschichtlich-mythologische Untersuchung," in: Mitteilungen Vorderasiatische Gesellschaft. Jahrg. 6. Berlin.

1903 Abraham als Babylonier, Joseph als Egypter. Der weltgeschichtliche Hintergrund der biblischen Vätergeschichten auf Grund der Keilschriften dargestellt. Leipzig.

1904 Die Weltanschauung des alten Orients. Leipzig.

1905 Zur babylonisch-assyrischen Geschichte. Leipzig.

English authorized translation and ed. by James Alexander Craig: The History of Babylonia and Assyria. London, Hodder \& Stoughton, 1907.

1906 Altorientalische Geschichtsaufassung. Leipzig.

1907 Die jüngsten Kämpfer wider den Panbabylonismus. Leipzig.

1913 Vorderasien im 2. Jahrtausend. Leipzig. 


\section{GEORG WISSOWA}

\section{Bibliography}

Kern, Otto, "Schriften von G. Wissowa," Jahresbericht über die Fortschritte der Klassischen Altertumswissenschaft. CCXLV (1934), 4. Abt., pp. 120-146.

\section{Main Publications}

1885 Religion und Kultus der Römer. (Handbuch der Klassischen Altertumswissenschaft. Bd. 5, Abt. 4). München, $1912^{2}$.

1893 Pauly's Real-Encyclopädie der classischen Altertumswissenschaft. Herausg. von G. Wissowa.

1904 Gesammelte Abhandlungen zur römischen Religions- und Stadtgeschichte. Ergänzungsband zu des Verfassers Religion und Kultus der Römer. München.

\section{GEORG WOBBERMIN}

\section{Bibliography}

In: Luther, Kant, Schleiermacher in ihrer Bedeutung für den Protestantismus. Georg Wobbermin zum 70. Geburtstag (27. 10. 1939), dargebracht von Kollegen, Schülern und Freunden. Berlin, 1939, pp. 578-588.

\section{Main Publications}

1896 Religionsgeschichtliche Studien zur Frage der Beeinfussung des Urchristentums durch das antike Mysterienwesen. Berlin, Ebering.

1902 Der christlische Gottesglaube in seinem Verhältnis zur heutigen Philosophie und Naturwissenschaft. Berlin, Alexander Duncker. Leipzig, Hinrichs, $1911^{3}$.

English translation from the third German edition by Daniel Sommer Robinson: Christian Belief in God. New Haven, Yale University Press, 1918. Japanish edition, Fukuoka, 1920.

1910 "Der gegenwärtige Stand der Religionspsychologie," Zeitschrift für angewandte Psychologie, III (1910), pp. 488-540.

1910 Aufgabe und Bedeutung der Religionspsychologie. Sonderausgabe aus dem Protokoll des 5. Weltkongresses für Freies Christentum und Religiösen Fortschrift. Berlin-Schöneberg, Protestantischer Schriftenvertrieb.

1911 Geschichte und Historie in der Religionswissenschaft. Utber die Notwendigkeit, in der Religionswissenschaft zwischen Geschichte und Historie strenger zu unterscheiden, als gewöhnlich geschieht. Adolf Harnack zu seinem sechzigsten Geburtstage. Tübingen, Mohr. 
1911 Monismus und Monotheismus. Vorträge und Abhandlungen zum Kampf um die monistische Weltanschauung. Tübingen, Mohr.

1913 Zum Streit um die Religionspsychologie. Berlin-Schöneberg, Protestantischer Schriftenvertrieb.

1915 "Die Frage nach den Anfängen der Religion in religionspsychologischer Bedeutung," Zeitschrift für Angewandte Psychologie, IX (1915), pp. 333390.

1913-1921 Systematische Theologie nach religionspsychologischer Methode. Leipzig, Hinrichs.

1. Die religionspsychologische Methode in Religionswissenschaft und Theologie. $1925^{2}$ with title: Einleitung in die systematische Theologie. Prinzipien und Methoden-Lehre in Hinblick auf ihre Geschichte seit Schleiermacher.

2. Das Wesen der Religion. 1925.

English translation by Theophil Menzel and Prof. Dr. Daniel S. Robinson: The Nature of Religion. With an Introduction by Prof. Douglas Clyde Macintosh, Yale University. New York, Thomas Y. Crowell Company, 1933.

Japanese edition: Fukuoka, 1937.

3. Wesen und Wahrheit des Christentums. 1925² $1926^{3}$.

1921 Religion. Die Methoden der religionspsychologischen Arbeit. Berlin, Urban Schwarzenberg. (Später in dem Sammelwerk von Emil Abderhalden: Handbuch der biologischen Arbeitsmethoden, Abt. VI, Teil C. Berlin, Urban \& Schwarzenberg, 1928.)

\section{WILHELM WUNDT}

\section{Bibliography}

Wilhelm Wundts Werke. Ein Verzeichnis seiner sämtlichen Schriften. Ed. by E. Wundt. München, 1927.

\section{Biography and Appreciation}

Hess, S., Das religiöse Bedürfnis. Eine kritische Studie anhand der Religionstheorie W. Wundts. St. Gallen, 1935.

Hoffmann, Arthur, Wilhelm Wundt. Eine Würdigung (by several writers). Herausg. von A. Hoffmann. (Beiträge zur Philosophie des deutschen Idealismus, Bd. 2. Schlussheft). Erfurt, 1922.

Petersen, P., Wilhelm Wundt und seine Zeit. (Frommanns Klassiker der Philosophie, Bd. 13). Stuttgart, 1925.

Sganzini, C., Die Fortschritte der Völkerpsychologie von Lazarus bis Wundt. (Neue Berner Abhandlungen zur Philosophie und ihrer Geschichte, Heft 2). Bern, 1913.

Trebitsch, Rudolf, Wilhelm Wundt's "Elemente der Völkerpsychologie" und die moderne Ethnologie. Leipzig, 1914. 


\section{Main Publications}

1873-1874 Grundzüge der physiologischen Psychologie. Leipzig, 1880² (revised edition, 2 vols.); $1902^{4}$ (completely revised with index made by W. Wirth, 3 vols.); $1908-11^{\circ}$ (revised edition, 3 vols.).

English translation from the fifth German edition, 1902, by E. B. Titchener: Principles of Psychological Paychology. London, Swan Sonnenschein, 1904.

1880-1883 Logik, eine Untersuchung der Principien der Erkenntniss und der Methoden wissenschaftlicher Forschung, 2 vols. Stuttgart. 1893-952; 1906$1908^{3} ; 1919-21^{4}$.

1885 Essays. Leipzig. $1906^{2}$ (mit Zusätzen und Anmerkungen).

1896 Grundriss der Poychologie. Loipzig. 19057; $1911^{10}$.

English translation with the cooperation of the author by C. H. Judd: Outlines of Psychology. Leipzig, Engelmann, 1897. 1902² (rovised English edition from the fourth revised German edition).

1900-1920 Völkerpsychologie. Eine Untersuchung der Entwicklungsgesetze von Sprache, Mythus und Sitte, 10 vols., Leipzig. See especially Vols. IV-VI: Mythus und Religion.

1910-1921 Kleine Schriften, 3 vols. Leipzig.

1911 Probleme der Völkerpoychologie. Leipzig.

1912 Elemente der Völkerpsychologie. Grundlinien einer psychologischen Entwicklungsgeschichte der Menschheit. Leipzig.

English authorized translation by E. L. Schaub: Elements of Folk Poychology. London, Allen \& Unwin; New York, Macmillan, 1916.

1913 Reden und Aufsätze. Leipzig.

1914 Sinnliche und Ubersinnliche Welt. Leipzig.

1920 Erlebtes und Erkanntes. Stuttgart. 